\title{
Sacrifice et homicide en pays massa (Tchad)
}

Sacrifice and homicide in Massaland (Chad)

\section{Françoise Dumas-Champion}

\section{CpenEdition}

Journals

Édition électronique

URL : http://journals.openedition.org/span/514

DOI : 10.4000/span.514

ISSN : 2268-1558

\section{Éditeur}

École pratique des hautes études. Sciences humaines

\section{Édition imprimée}

Date de publication : 30 mai 1981

Pagination : 175-193

ISSN : 0294-7080

\section{Référence électronique}

Françoise Dumas-Champion, « Sacrifice et homicide en pays massa (Tchad) », Systèmes de pensée en Afrique noire [En ligne], 5 | 1981, mis en ligne le 04 juin 2013, consulté le 02 mai 2019. URL : http:// journals.openedition.org/span/514 ; DOI : 10.4000/span.514 


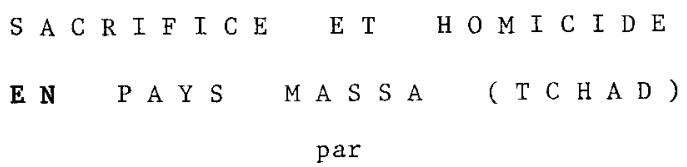

Dans un prëcélent article (1), nous avons souligné la distinction opérêe par les Massa sur le choix des victimes sacrificielles. Les animaux qui peuvent être mis à mort -- volailles, ovins, caprins et bovins -- s'avèrent être des bêtes dénuées de capacités vengeresses à la différence du chien qui, doté de caractères humains, possède un pouvoir vengeur appelè tokora et n'est pas, de ce fait, égorgé.

Qu'en est-il de ce tokora pour que les Massa justifient de son existence pour faire des animaux sacrificiels qui en sont pourvus, des victimes non sanglantes ? C'est dans le contexte de l'homicide que nous retrouverons toute 1 a portêe de cette notion. Le meurtre, déclencheur du tokora, nous confrontera aux rituels qui entourent 1 'homicide et aux interrelations du meurtrier et de sa victime. Nous transposerons ces rêsultats -- en ce qu'ils peuvent être révêlateurs de 1'impact d'une mise à mort -- dans le contexte sacrificiel.

I. Les mituels liés à l'homicide.

L'homicide détermine des conséquences de trois ordres pour le meurtrier. Quelles que soient les circonstances de son acte, qu'il ait tué

(1). Le sacrifice comme procès rituel chez les Massa (Tchad), Systèmes de pensée en Afrique noire, cahier 4, Le sacrifice III, 1979, pp. $94-115$. 
un ennemi à la guerre, au cours d'une expédition de vengeance, ou qu'il ait tué un parent, le meurtrier sera en proie au pouvoir vengeur de sa victime, à la vengeance des parents du mort s'il lui était étranger et à la poursuite de la Terre, Nagata, si le meurtre a eu lieu sur son propre territoire.

S'il advient, au cours d'une dispute, qu'on blesse à mort un parent, c'est la consternation, la torpeur. On dira du criminel qu'"il n'a plus de tête". Un parent, c'est un autre soi-même et un tel acte ne peut être commis que sous 1 'empire de la dêmence. "Les chenilles ne mangent pas les feuilles sur lesquelles elles se tiennent", dit un proverbe massa, à propos d'un tel fait. Autant I'homicide d'un parent est un évênement dramatique autant celui d'un étranger est valorisé. Si l'opposition aux ennemis ne s'exprime pas chez les Massa, comme chez les Mesmé du Sud du Tchad, par la nécessitê de tuer un homme pour acquérir une épouse, les guerriers massa, capables de tuer, occupent une place privilëgiée au sein du farana, la communauté de base. Ils assurent, par leur courage, la cohésion et l'unité du groupe. A la différence d'autres populations qui utilisent le corps de leurs victimes tuées à la guerre en guise de trophées ou pour la fabrication de médecines, les Massa abandonnent généralement le cadavre sur place. Tout au plus, chercheront-ils à le jeter dans le fleuve afin que les parents des tués ne les retrouvent pas. Autrefois, il leur arrivait cependant de décapiter les corps et certains rapportaient les têtes qu'ils conservaient dans leur case. I1 ne semble toutefois pas que ces faits aient été fréquents et qu'on puisse attester l'existence d'un culte de ces trophées. Mais de tels actes dénotaient le courage du guerrier capable de vaincre la peur attachêe au cadavre.

En effet, lorsqu'on découvre en brousse ou au bord de l'eau le corps d'un étranger, on s'enfuit sans même le toucher, de crainte d'être pris par la puissance responsable de sa mort. Si les parents de la victime recherchent le cadavre, on leur donnera quelques indications, sans toutefois préciser le lieu exact. On dit tout simplement qu'il y a "une chose" en brousse ou à proximité du fleuve. Nommer la "chose" s'avèrerait dangereux pour I'homne qui la désigne. Les proches du mort viendront le récupérer. Mais ce sera la tâche des hommes adultes; les jeunes devront éviter de le voir ou de le toucher. 
A. Ensevelissement de la victime.

Si 1'homme a été tuê par un étranger -- ce que 1 'on suppose lorsqu'on ne connaît pas le coupable --, on ensevelit la victime en lui donnant les moyens de se venger. On lui remet une arme. Alors que les autres morts sont enterrés couchés, dans la position du repos, l'assassiné est accroupi, les yeux ouverts, le bras gauche attaché à la taille, tandis que le droit est relevé au-dessus de la tête, l'index pointé vers le ciel de manière à y attacher la lame d'une sagaie, l'extrémité du doigt et l'arme émergeant de la tombe, une fois le corps enseveli. Comme autre mode de vengeance, on introduit dans la bouche de la victime un margouillat qu'on vient de décapiter. Le lézard gris qui, tout comme 1'homme, a le "sang amér", dispose d'un pouvoir vengeur, appelê également tokora. Ici, on utilise des médecines pour diriger le pouvoir maléfique de l'animal vers le criminel. L'addition des deux tokora doit mener $1 \mathrm{a}$ vengeance à exécution.

Si la victime est une femme, elle ne sera pas vengêe. Le cadavre n'est done pas traité pour qu'il puisse assurer sa propre vengeance. Il ne reçoit ni lance, ni margouillat come le corps d'une victime apparentêe à son meurtrier.

B. Réclusion du meurtrier.

Après le meurtre, le tueur accomplira une retraite destinée à le protéger du pouvoir vengeur de sa victime -- que le cadavre ait été enseveli ou qu'il n'ait pas été retrouvé. Il s'isole alors durant trois (2) jours, aidé par 1'un des siens, un ancien tueur, qui le guidera dans le déroulement du rituel. En proie à la vengeance des parents de la victime, le meurtrier reste à proximité de son farana (3). Il doit simplement se cacher sous un arbre, à l'abri des regards. Personne, du reste, ne cherchera à le rejoindre ni à lui parler, car tout contact avec lui suffit à contracter le tokora. Cette période de réclusion, en

(2) Trois : chiffre masculin.

(3) farana : communauté de base. 
retrait de toute habitation, est essentiellement destinẻe à assurer la protection de son entourage. Le tokora qui poursuit le coupable ne distinguerait pas le meurtrier d'un simple passant qui aurait eu le malheur de lui adresser la parole. Tout comme le tueur, celui-ci devrait se plier au ritue 1 du tokora.

Si 1 'homme a tué un parent, il ira pleurer le mort en compagnie des autres membres de sa famille, mais il quittera le lieu funèbre avant qu'on comnence à creuser la tombe qu'il ne doit pas voir sous peine de mourir -- "c'est comme si la tombe était pour lui", disent les Massa. Cette fois, il partira loin de sa terre, dans un endroit où il ne risque pas d'entendre le tambour qu'on frappe en 1 'honneur du mort. Il mourrait s'il entendait le son du tambour qu'on dit être "la voix de la victime qui 1 'appe11e".

Que 1'assassiné soit un étranger ou un parent, le tueur est soumis au même type de rituel. Toutefois, un homme qui a tué un ennemi tentera, à la nuit tombée, une expédition chez sa victime afin de prélever que1ques brins de paille au toit de sa case. Avec cet objet appartenant au mort, il réussit à "maîtriser" le tokora. On dit alors que "le meurtrier a pris 1'âme du mort". Dès lors, le tokora n'est plus à même d'identifier le tueur puisque celui-ci s'est assimilé à sa victime. Malheureusement pour lui, il s'avère extrêmement difficile d'obtenir cette paille. La famille monte, en effet, une garde vigilante et exécuterait quiconque tenterait de s'en emparer.

Avant de s'isoler, le meurtrier se rend chez un préparateur de "médecine", sa-ma-guna, qui lui remet des médecines, guna tokora, faites de tiges de subulza (Cissus quadrangularis) que le tueur porte à sa bouche et avec lesquelles il se frictionne le corps afin de s'imprégner de cette plante dont l'amertume est censée repousser le tckora. Puis, il s'attache le subuzla autour de 1a tête, du cou et des poignets. I1 se recouvre ensuite le corps de cendre, prélevée clandestinement dans 1a cuisine d'une vieille femme. Il s'agit de la cuisine d'une femme âgée qui ne procrée plus, car le tokora est suffisamment dangereux pour atteindre la propriétaire du foyer ou sa descendance. Recouvert de cendre, il est comme mort. En se donnant l'apparence d'un cadavre, les Massa disent qu'il cherche à tromper la victime, qui, ainsi, ne s'acharnera plus sur lui. Notons que les deuilleurs qui se barbouillent également de cendre utilisent les mêmes artifices aux mêmes fins. L'identi- 
fication du meurtrier aux deuilleurs ne s'arrête pas 1à. Tout comme le veuf pendant sa réclusion, le tueur doit se tenir éveillé pour éviter que la victime le prenne pendant son sommeil. Les Massa disent que "celui qui fait le tokora est comme celui qui suit le rituel de deuil". Les veufs craignent, en effet, le yowna du mort qui vient prendre son conjoint pour poursuivre le chemin qu'ils ont entrepris ensemble. Mais, à la différence du tokora, le yauna est dénué de toute forme de vengeance. Il est comme l'émanation du lien créé par l'accouplement. C'est la force produite par l'union du couple qui subsiste au-delà de la mort. Tout se passe comme si le mort n'avait de cesse de rejoindre son conjoint et de tenter de 1'entraîner dans la mort.

Le meurtrier est soumis à un régime alimentaire spécifique qui complète son traitement extérieur et assure sa protection. Alors qu'il s'est donné préalablement 1 'apparence d'un mort, il saupoudre maintenant de terre sa "boule" de mil pour prouver qu'il est bien mort. Et, tout en absorbant la première bouchée, il se plaint de son sort en adressant une prière à la Terre : "Nagata, pardonne-moi pour avoir répandu le sang humain, mais ce n'est pas moi qui l'ai voulu, c'est Matna" (4). Il consomme également des aliments "morts", comme du poisson sec, que les Massa opposent au poisson frais ou à la viande fraîche qui sont "vivants" et que la cuisson ne tue pas. Alors qu'au premier stade de sa réclusion, le meurtrier s'était imprégné le corps d'amertume, il absorbe maintenant une sauce à l'oseille dont l'acidité joue encore le rôle d'un révulsif. L'alimentation du tueur, à base de produits "morts" ou qui "repoussent", assure son traitement interne. Sa protection se trouve encore renforcée par une fumigation des mêmes plantes amères, subulza, qui brûlent en permanence pendant son séjour.

Après ces trois jours de réclusion, le meurtrier retourne voir le sa-ma-guna qui le conduit dans un marigot où il le lave. Ce rite final, qui redonne au tueur son apparence de vivant en le débarassant des traces qui le faisaient ressembler à un mort, marque la fin de son isolement et de l'emprise dangereuse du tokora. Le sa-ma-guna lui remet ensuite d'autres médecines qui devront le protéger durant toute sa vie.

(4) Matna : puissance responsable de l'homicide. 
II en portera en permanence sur lui -- et il en plante dans son champ ou à proximité de son habitation.

Le quatrième jour, le tueur rentre chez lui. Si sa victime est un ennemi, il cherchera à la tuer une seconde fois. Il place alors une nasse à l'entrée de sa case pour attraper l'esprit du mort qui vient pour le saisir. Averti, dit-on, par le bruit que fait "1'âme" prise au piège, il donne des coups de lance dans la nasse. Les vieux racontent qu'à ce moment, ils voient du sang couler. La victime tuée "corps et âme", l'assassin est libéré définitivement. Cependant, les Massa ont 1 'habitude de dire qu'un tueur, guerrier ou criminel, mourra toujours dans des circonstances pénibles, en proie au tokora. Il attrapera la gangrène ou la lèpre et sera enterrë comme l'assassiné selon les normes d'ensevelissement réservêes aux victimes des morts brutales, watzawatzana.

c. Vengeance des parents de la victime.

Trois jours après le meurtre, la famille de la victime et ses alliés manifestent leur volonté de vengeance en portant dans le camp du meurtrier un objet appelé tomkolta ainsi que la lance de la victime. Le tomkolza, composé de paille grossièrement tressée puis roulée comme un tapis à l'intérieur duquel on insère des cendres provenant du foyer central de l'habitation ainsi que des crottes de chien, représente 1 'enclos et ses membres. Les cendres sont utilisées comme symbole du foyer et les crottes manifestent la présence du chien qui veille la nuit, couché près du feu qui couve. On dit de lui qu'il est en contact avec les esprits et les ancêtres qui viennent rôder. Il symbolise la paix de l'enclos. En abandonnant le tomkolza chez le meurtrier, la famille de la victime effectue symboliquement le retour du meurtre dans le camp adverse. La présence du tomkozza dans le territoire ennemi doit produire un nouvel homicide. L'enjeu qui est d'importance engage inêvitablement la riposte de l'ennemi. Aussi, avant d'entreprendre une telle dêmarche dans le camp de la victime s'assure-t-on qu'on est en position de force.

Le rite commence dans la cour de l'enclos où tous les membres de la famille sont réunis. Les hommes sont armés de leur bouclier et de leur sagaie. Ils viennent de retirer du cadavre la lame de sagaie qui 
émergeait du sol. A ce moment, c'est comme si le mort se levait luimême pour se venger. L'homme le plus fort de la famille, désigné pour porter le tomkolza, tient dans la main droite la sagaie du mort. Rassemblés comme pour partir en guerre, les hommes sortent ensemble de 1 'enclos pour se diriger vers le territoire ennemi. Les membres de leur farana (5) et d'autres farana alliés se joignent alors à eux. Les femmes, à 1'arrière, excitent les hommes par leurs cris. Le camp ennemi qui prévoit 1 'affrontement se prépare à 1 a guerre. Le guerrier muni de la lance du mort cherche à atteindre un parent du meurtrier qui mourra du tokora même si 1 'arme ne I'atteint pas mortellement. I'homme qui a la charge du tomkolza doit donc être suffisament bien caché par d'autres guerriers afin que le camp adverse ne puisse repérer 1 'arme de la victime qui ne devra pas tomber à terre sans avoir touche 1 'ennemi, sinon le tokora resterait dans le camp de la victime.

Lorsqu'on ne connaît pas le responsable du crime, la famille dépose le tomkolza en brousse, loin des habitations et elle plante la lance du mort en terre, tout en proférant 1a menace suivante: "Que le meurtrier périsse !". Si les capacités guerrières du farana de la victime s'avèrent insuffisantes pour risquer 1 'affrontement, on dépose le tomkolza à la limite du territoire ennemi, tandis que, de nuit, deux ou trois agnats du mort se rendront chez le meurtrier sans se faire remarquer, ils ficheront la lance dans le toit de sa case afin de retourner le tokora sur le tueur. Pour l'homicide d'un parent, la famille va déposer le tomkolza à la limite du farana, non loin des habitations. Le tomkolza, alors dénué de sa fonction vengeresse, doit permettre 1 'êloignement de la force malfaisante du crime dont on tient seul Matna responsable.

D. Le meurtrier et sa terre.

C'est seulement lorsque $1^{\prime}$ homicide a lieu sur la terre du meurtrier qu'un sacrifice doit la "rafraîchir". Un mois environ après son crime,

(5) farana : communauté de base et unité de combat. 
le tueur remettra une chèvre au chef de terre qui l'imolera à l'entrée de son enclos. Bien que le meurtrier n'assiste pas au ritue1, l'officiant prononcera une prière en sa faveur : "Nagata, laisse le meurtrier en paix, donne-lui la fraîcheur". Le sang de l'animal qui s'écoule à terre aura un double effet. C'est un sacrifice de type porra (6) à 1 'égard du tueur qui devra rafraîchir son corps, rendu chaud par 1'homicide et de type divinna (7) pour la terre qui, également chaude, doit être rafraîchie. On procède comme pour chaque sacrifice à la terre. L'animal est découpé sans être dépecé, la peau n'est pas conservée afin d'êpargner son utilisateur qui risquerait d'être pris par Nagata. La viande est distribuée entre les différents lignages (8) habitant sur cette terre, sans qu'il soit tenu compte du nombre de ses membres, qu'ils soient dix ou une centaine. L'aîné du lignage partage ensuite le morceau reçu entre toutes les familles. Le chef de terre, responsable du sacrifice, conserve toutes les parties internes de la dépouille ainsi que la tête. II reçoit, en plus, comme chacun, un morceau de viande. Seuls le meurtrier et sa famille n'obtiennent aucune part. Il est de règle, dans les sacrifices à la terre,que celui qui fournit 1'animal ne consomme jamais sa chair. Les Massa l'expliquent comme un moyen de mettre un terme à l'insatiabilité de Nagata qui reviendrait réclamer d'autre sang. Chaque famille s'occupe donc, pour son compte, de la cuisson de sa part de viande. Elle peut la faire griller, dans la mesure où elle n'est pas concernée par le sacrifice, mais elle ne doit, à aucun prix, introduire la viande dans l'enclos, sous peine d'y faire pénétrer Nagata et les maux qu'elle causerait. Par contre, la femme du chef de terre met la viande sacrificielle à bouillir. La cuisson achevée, le prêtre prélève une parcelle de chaque viscère, de la langue et du gigot qu'il a conservés et offre cette "bouche de la viande", en réitérant la prière qu'il a adressée préalablement à Nagata. Les autres familles doivent attendre cette offrande pour consommer la part qui leur revient.

(6) Pour des indications générales sur le porra, cf. F. Dumas-Champion, op. cit. pp. 95-101.

(7) Sur le divinna, op. cit. pp. 106-107.

(8) En moyenne, trois lignages cohabitent sur une même terre. 
Bien que la terre soit tout aussi "chaude" si le meurtre a été commis sur la terre de la victime et non sur celle du meurtrier, les parents de l'assassiné n'effectueront aucun sacrifice pour la "rafraîchir". Elle le sera par la vache que le prêtre de la terre immolera à 1 'occasion des semailles. La famille du mort, qui a déjà subi une perte, n'a pas, disent les Massa, à fournir une bête sacrificielle. Par contre, le tueur qui a rompu l'interdit condamnant 1 'homicide sur sa terre, doit "racheter sa faute" par le don d'une chèvre et éviter ainsi la poursuite de Nagata. Dans la mesure où c'est sa propre terre qui l'attaque, $i l$ ne risque rien lorsqu'il n'habite pas la terre qui a reçu le sang du crime. Le lieu où le sang a été versé devient un terrain "chaud" qu'on abandonne s'il était livré préalablement à la culture. Ce champ ne sera plus jamais cultivé.

E. Sacrifice des "mains réunies".

Environ trois mois après l'enterrement de la victime, un sacrifice appelé tap bam'na, "les mains réunies", doit ressouder les liens des deux familles apparentées, mais sêparées par le meurtre. En effet, dès que la tombe avait été creusëe, les parents du meurtrier ne pouvaient plus pénétrer dans 1 'enclos de la victime, par crainte du tokora. Le sacrifice d'un taureau, veau ou bouc, apparaît comme le seul moyen de mettre un terme au tokora et de permettre aux deux familles de renouer contact. Elles contribuent toutes deux à l'achat de l'animal.

La victime, tenue par le cou, est sortie de l'enclos du mort pour être sacrifiée à l'endroit où le tomkolza a été déposé. L'honme le plus âgé vivant dans l'enclos de la victime joue le rôle de sacrificateur. I1 invoque I'ensemble des puissances : "Lawna (Dieu), Nagata (Terre), Fuliana (l'ensemble des autres divinités), réunissez-nous les mains". L'animal est découpé avec la peau. On vide soigneusement les viscères, puis on met le tout à bouillir. Rien ne devra être jeté. On plonge même quelques instants les cornes de la bête pour signifier qu'elles ont été cuites comme le reste. A côté, on prépare la farine apportêe par les deux familles. Les poteries utilisées pour la cuisson ainsi que le couteau sacrificiel seront abandonnës sur place. La viande une fois cuite, on en prêlève "la bouche" en coupant une parcelle de chaque partie de 1 'animal : entrailles, langue, gigots..., qu'on met dans un morceau de 
poterie avec un peu de "boule" écrasée. On dépose le tout à côté du tomkolza en répétant la prière précédente. Seuls les membres des deux familles consomment sur place la viande et la boule que le sacrificateur distribue. Il est alors possible de retirer la peau restée attachée à 1a chair, mais, en aucun cas, il ne faut casser les os. La victime symbolisant 1 'union des deux familles, en briser les os signifierait leur séparation. Le meurtrier, qui avait quitté sa terre après la mort de son parent, revient pour participer au rituel. Il reçoit la tête de I'animal, qu'il doit réussir à manger sans la casser. La tête, dit-on, contient la force vitale de $l^{\prime}$ animal dont le meurtrier a besoin pour reconstituer sa propre tête, atteinte par son acte criminel.

- Pour clore la cérémonie, le sacrificateur bénit l'assistance, ycom nina, en l'aspergeant d'eau dans laquelle se trouvent les poissons préférés de Mununda (9), dakna. Cette bénédiction a pour but de rafraîchir I'assistance qui quitte les lieux sans se retourner. Le meurtrier regagne sa terre d'exil, où il vivra encore une année, mais il pourxa désormais rendre visite à ses parents, sans toutefois voir la tombe de sa victime. Par contre, les deux familles pourront se rendre 1'une chez 1 'autre et prendre leur repas en commun.

F. Sacrifices du meurtrier.

Un an après le meurtre, pour assurer sa protection et celle de sa famille, le meurtrier accomplit un sacrifice, divinn watzana, qu'il renouvellera chaque année et que ses fils continueront après sa mort, dans la mesure où le tokora s'attaque à la descendance du coupable. La première fois, la victime sacrificielle diffère de celles qui suivront. On commence par sacrifier un bouc à l'intérieur de l'enclos. L'aîné de la famille fera office de sacrificateur. Il prononce une prière pour que le tokora ne revienne pas. L'animal est découpé avec la peau. Puis, avant d'être mis à bouillir, le meurtrier prélève la tête du bouc ainsi que les quatre pattes qu'il place sur un petit noulza, brise-vent fabriqué pour 1 'occasion qui protège généralement l'ouverture des habitations

(9) Mununda : génie de l'eau. 
et qui est ici censé empêcher le tokora de pénétrer dans la case. I1 porte alors son sacrifice à l'endroit où a été déposé le tomkolza, ou tout simplement en brousse si le tomkolla a été renvoyé en territoire ennemi. C'est la part pour Matna et Bagawna, les fuliana de la brousse. A son retour, avant de commencer le repas, le sacrificateur prélè̀ve la "bouche" de la viande, qu'il jette pour Matna dans la cour de 1'enclos. L'année suivante, à la saison froide, le meurtrier doit offrir en sacrifice un couple de chiens ainsi qu'un oeuf qu'il part déposer en brousse. Les chiens sont laissês libres mais on choisit de jeunes chiots pour qu'ils soient incapables de retrouver leur domicile. Ce qui signifierait le retour du meurtre et ses conséquences qu'on avait pris soin de chasser. A défaut de chiots, il est possible de tresser un semblant de chien en paille. Le chien qui, nous 1 'avons vu, est 1 'animal le plus proche de 1 'homme, a, comme lui, le "sang amer". I1 est simplement remis entre les mains des génies de brousse sans être mis à mort car, doué d'un tokora comme 1 'home, il poursuivrait son tueur et ferait périr sa descendance. Abandonnés en brousse, les chiots sont voués à une mort certaine. Il ne s'agit pas d'un sacrifice sanglant qui irait ici, selon la logique massa, à 1 'encontre des bienfaits recherchés dans ce sacrifice.

G. tokora du chien et du margouiziat.

Les chiens ne sont donc jamais égorgés au cours de rituels sacrificiels, mais $1^{\prime}$ effet de leur tokora est parfois dénoncé lors d'une séance divinatoire. La maladie d'une épouse en grossesse ou celle d'un nourrisson peut être interprétée come l'expression de la vengeance d'un chien que le mari aurait tué dans son enfance. Le devin prescrit alors un sacrifice en 1 'honneur du chien, êlevé au rang de divinité. Le mari ira dêposer sur le chemin un os brisê en sept morceaux baignant dans un fragment de poterie rempli d'eau, puis il jettera deux criquets derrière la haie d'épines qui entoure l'enclos. Si malgré ce sacrifice, le nouveau-né meurt, le prochain enfant recevra le non de chien : Dimadi pour un garçon, et Dia pour une fille. Les Massa indiquent une possibilité de supprimer l'effet du tokora en comptant les dents du chien. Dès qu'on a terminé le compte des dents sans se tromper -- on ne peut compter qu'une seule fois -- on se trouve libéré du tokora. 
Un autre animal au "sang amer", le margouillat, possède, lui aussi, un tokora qui s'attaque au pouvoir de procréation de son tueur. L'enfant d'un parent coupable d'avoir tué ce lézard gris recevra, lui aussi, pour nom celui de 1 'animal. Une femme enceinte qui écraserait une femelle margouillat pleine risque d'avoir des problèmes au moment de $I^{\prime}$ accouchement: Elle-même ou son enfant peuvent mourir, tout comme la femelle a përi avec sa descendance. L'enfant et la mère courent les mêmes risques lorsque le père a tué plusieurs de ces animaux dans son enfance. C'est donc, souvent, un fait amnésié que le devin rappelle.

Quelques jours après sa naissance, le nouveau-né peut attraper "la maladie du margouillat". Il commence à baver, sa peau se desquame comme celle d'un saurien et des taches blanches apparaissent sur son corps. Pour tenter d'annuler le tokora, un sacrifice est dédié au margouillat qui, d'ancienne victime et de simple lézard, est élevé au rang de fuzza, puissance supra-naturelle. Le rituel sacrificiel doit se faire en présence de deux margouillats, mâle et femelle, capturés et attachés pour la circonstance puis enduits de terre ocre, čna. Le lendemain matin, à l'aube, le sacrificateur assomme deux poussins devant l'entrée de I'habitation réservée à son épouse. Trop petits pour être consommés, sa femme prépare une cuisine simulacre en les mettant à bouillir quelques instants dans une petite poterie et en allumant le feu sous une autre pour la farine. Le mari prélève ensuite la "bouche de la viande" et la dépose avec deux criquets devant. les margouillats qu'il libère. Le sacrificateur, en compagnie de ses cadets, en $I^{\prime o c c u r e n c e ~ d e s ~ e n f a n t s ~}$ puisqu'il n'y a rien à manger, portent symboliquement une bouchée à leur bouche. La femme et le nourrisson sont exclus de ce repas simulacre. Si l'enfant guérit à la suite du sacrifice, on le nomera "margouillat" : Iudongola pour un garçon, Hudongolda pour une fille. S'il meurt, c'est à leur prochain enfant que les parents devront donner ce nom. Les Massa choisissent gënéralement les noms de leurs enfants en fonction des traits marquants qui ont prêcédé la naissance. Cette attribution peut être comprise en fonction du principe d'échange inhérent au système sacrificiel massa. Tout se passe comme si on "donnait" son enfant en le vouant à la puissance en échange de sa vie sauve. 
II. Le meurtrier et sa viatime, le sacrifiant et sa viatime.

Du déroulement du rituel transparâ̂t la relation étrange qui se trame entre le meurtrier et sa victime. Le tueur s'assimile à celui qu'il vient de tuer. Il traite son corps extérieurement et intérieurement comme si'il était mort. Certes, les Massa disent qu'il se fait "passer pour mort". pour détourner la vengeance de sa victime, mais que penser alors du comportement d'un homme meurtrier d'une femme qui $s$ 'habille comme sa victime, revêt la ceinture pelvienne, se pique les lèvres de paille pour simuler les labrets, et va, pendant la période du tokora, s'occuper à des tâches féminines comme piler le mil ? Si 1'on suit le raisonnement massa, faire le mort devrait suffire à détourner le tokora, mais ce pouvoir vengeur ëmanant d'une victime de sexe opposé est, disent-ils, encore plus dangereux. L'usage de cet accoutrement se présente alors comme la meilleure façon de "tromper" sa victime. Tout se passe pourtant comme si le meurtre contraignait le meurtrier à perdre sa propre-identité. Doit-il prêter son corps à sa victime pour prolonger quelque temps la vie qu'il lui a retirée brutalement ? L'acte criminel qui, cependant, marque une coupure crée, de fait, un lien irrémédiable entre les deux partenaires. La relation de vengeance qui les unit va faire en sorte que le tueur devienne à son tour une victime. Tout comme elle, il meurt de mort violente : watza-watzana. Une fois de plus, le sort réservê au meurtrier l'identifie à sa victime. Ils ne sont rien 1 'un sans 1 'autre et apparaissent comme interchangeables. De même que le guerrier yanomami (10) ayant tué un ennemi, le meurtrier massa, à la suite de son acte, est pris de vertiges, sa vue se trouble, son corps devient mou, il a "mal aux articulations". Plutôt que l'expression d'un remords qui rongerait le tueur et l'affaiblirait physiquement, cet état apparaît comme l'expression de la symbiose du meurtrier et de sa victime, de l'état d'indistinction où se perdent les limites mêmes de leurs corps respectifs. Les symptômes que ressent le meurtrier traduisent les "réactions" de sa victime : s'il ne ressent rien, c'est que sa victime a recouvré la santé. Cette relation est bien celle qu'on retouve dans le processus sacrificiel entre le sacrifiant et sa victime puisque l'animal est substitué au corps du sacrifiant pour

(10) Ettore Biocca, Yanoama, Paris, Plon, pp. 66-69. 
mourir à sa place. Si les divinités massa acceptent le sang d'une bête en êchange de celui d'un homme, c'est que le sacrifiant et sa victime font corps et sont interchangeables. Un individu n'est rien sans sa bête sacrificielle; sans elle, il meurt, pris par la puissance.

Tout se passe comme si le sacrifiant puisait sa vie dans celle de sa victime. Il est "rafraîchi" ou reconstitué grâce à elle. Elle lui donne son sang ou sa tête, comme le montre le sacrifice "des mains réunies", oû le meurtrier doit assimiler la tête de l'animal pour retrouver'sa propre tête que l'acte criminel lui avait fait perdre.

Le cadavre de la victime, comme le corps vivant du meurtrier, sont 1 'objet des mêmes évitements. Toutefois, on n'encourt pas le même risque à leur contact. Le cadavre, pense-t-on, appartient aux puissances responsables de la mort ; le meurtrier n'est qu'un exécutant. Un corps flottant dans le fleuve sera la propriêté de Mununda, génie de l'eau, tandis qu'il appartiendra à Matna ou à Bagawna (11) s'il est découvert en brousse. Le corps d'un homme tué dans son enclos appartiendra encore à Matna, responsable du meurtre. Donc, toute tentative pour retirer le corps, ou simplement lui manifester des signes d'attention, revient, aux yeux des Massa, à s'interposer dans le dessein des divinités qui en ont fait leur proie. Toucher ou nommer le cadavre, c'est une façon de s'approprier ce qui appartient aux puissances. C'est risquer d'encourir leur sanction, d'être "pris" à son tour et d'en mourir. Le danger est tel que 1 'homme qui aura, par exemple, enterrê un noyé et qui aura dû déplacer le corps -- manifestant par ce déplacement comme une reprise du cadavre, bien que le corps soit toujours enseveli à proximité de l'eau pour montrer justement qu'il appartient au génie -- accomplira, tout comme le meurtrier, un divinn watlona (12), sacrifice de mort violente, adressé à. Mununda, afin d'assurer sa protection.

Décrocher le cadavre d'un pendu est peut-être encore plus dangereux,

(11) Bagawna : génie protecteur de la brousse et de la faune.

(12) divinn watlana : sacrifice identique à celui effectué par le meurtrier, comprenant la même prière, puisque le génie met en danger la vie et le pouvoir de procréation du contrevenant. On offre en plus à Mununda des graines qui produisent en grande quantité comme le pénicillaire, Ceina, le sésame, dak̉ lawna, et l'éleusine, dana. 
si l'on juge le rituel sacrificiel qui va permettre la dépendaison. Le moment fatidique pour le proche parent (13) chargé de l'affaire est celui où il doit rompre la corde qui retient le corps. Pour faire croire à Matna qu'on n'a pas décroché le cadavre, on lui substitue une brebis (14) en pendant 1 'animal à la place du mort. Le mouton attachè à la même corde va mourir étranglé comme le pendu. La pendaison de 1a brebis est un sacrifice de type porra, destiné à "rafraîchir" le parent qui coupe la corde. Avant de pendre 1'animal, le sacrificateur indique dans sa prière à Matna qu'il n'a pas enlevé "sa chose". Un second sacrifice (porra) de mouton aura lieu après l'enterrement. Cette fois, le responsable mettra les pieds dans le sang. Cette protection devra être renouvelée chaque année par un divinn watzana dédié à Matna.

Que celui qui déplace un cadavre soit soumis au même rituel sacrificiel qu'un meurtrier apparaît toutefois surprenant. Pourquoi ces deux actes -- prélever un corps et retirer une vie -- sont-ils traités sur le même plan alors que, de toute évidence, les Massa les diffêrencient ? En fait, le mort, comme le meurtrier, apparaissent tous deux comme les victimes de Matna, qui a utilisé l'un pour tuer l'autre. Le cadavre de la victime, comme le corps vivant du meurtrier, sont tous deux la propriété de la puissance. Aussi, le déplaceur de cadavre qui retire au dieu sa proie tombe-t-i1, à son tour, sous son emprise. Le meurtrier, proie vivante de la divinité, cherche, tout comme le preneur de cadavre, à se libérer de cette emprise qui se manifeste par le pouvoir vengeur de sa victime. En dédiant un sacrifice à Matna, il cherche à se libérer du tokora manoeuvré par la dite-puissance à l'origine du meurtre. L'un et 1 'autre se trouvent donc contraints au même sacrifice qu'ils renouvelleront chaque année.

Bien que le meurtrier soit aussi la propriété de Matna, ce n'est pas directement en tant que tel qu'il est l'objet d'évitement, mais

(13) Si le suicidé n'a pas de cousin proche, ce rôle sera tenu par le neveu utérin; s'il n'a aucune famille, on laisse le cadavre suspendu, puis, de l'extérieur, on écroule le toit et les murs de la maison qu'on entoure d'épines pour éviter le contact du bétail, des chiens ou des charognards. Le mort ne fera 1 'objet d'aucun rite funéraire.

(14) On choisit toujours un animal de sexe opposé au pendu. 
dans 1a mesure où il transmet le tokora. Il suffit de le toucher, de lui parler ou même de le voir pour être contaminé. Comme il souille tous les endroits où il passe, il ne peut s'isoler qu'à l'écart des habitations, caché sous un arbre, pour ne pas avoir à détruire 1a case qu'il utiliserait. Toutes les sociétés mentionnent la souillure de I'homicide ou du chasseur qui motivent sa réclusion. I1 faut que le meurtrier se décharge de toutes ses impuretés pour reprendre le cours normal de la vie. Mais ce pouvoir de contamination fait ici apparaître l'incapacité du tokora à reconnaître son tueur, puisqu'un simple passant qui s'adresserait au coupable serait impliqué au même titre qu'un criminel. La communication par la parole, le toucher ou 1a vue lie les acteurs jusqu'à leur conférer les mêmes caractéristiques. Tout se passe comme si de la parole, du contact ou de la vue du meurtrier émanait une part de sa personne aussitôt assimilée par celui qui l'écoute, le touche ou le voit. D'autres faits montrent qu'un objet prêlevé sur la personne 1 'exprime en propre. Ainsi prélève-t-on sur le corps d'un noyé un morceau de ses vêtements ou une rognure d'ongle pour les enterrer à 1 'emplacement même où le cadavre devrait reposer, devant l'entrée de sa case. Cette partie du corps est traitée comme le serait l'ensemble puisque ce lieu est considéré comme la tombe réelle. C'est à cet emplacement même qu'on sacrifiera le mouton des funérailles et non sur 1a tombe qui contient le cadavre. C'est ainsi également qu'il suffit au meurtrier de tenir un brin de paille appartenant à la case de sa victime pour brouiller les pistes du tokora (15). Ce pouvoir vengeur semble une force aveugle ne pouvant se repérer qu'aux signes ou aux marques attachês à la personne et dénué de tout sens moral. A aucun moment du reste les Massa ne justifient le rituel du tokora comme exprimant un remords.

Si les mêmes interdits d'évitement frappent la victime comme son meurtrier, des faits montrent qu'un sacrificateur ou un sacrifiant est, lui aussi, objet de souillure. Un home qui parlerait à un passant alors qu'il se rend en brousse, au bord de l'eau ou à la croisée des chemins pour sacrifier impliquerait l'inconnu dans son sacrifice. De même que le meurtrier contamine son interlocuteur, en lui parlant, de même, par sa parole, le sacrifiant communique au passant toutes les impuretés

(15) Cf. plus haut p. 178. 
qu'il a symboliquement draguees de son enclos, avant d'en sortir, pour les évacuer sur un autre territoire. Les sacrifices qui s'effectuent à I'extérieur de 1 'habitation commencent à la porte, où le sacrifiant prononce sa prière. Entre sa sortie et 1 'endroit éloigné où il dépose la matière oblatoire jusqu'à son retour au sein de l'enclos qui marque la fin du rituel, il effectue un parcours qui est une séquence à part entière du procès sacrificiel. Le sacrifiant adopte du reste un comportement spêcifique. Tête baissẻe pour s'isoler du monde extérieur, il tient son offrande dans la paume des mains ou il conduit par le cou l'animal qu'il va sacrifier. Il est alors en relation avec la puissance à laquelle il vient de s'adresser et dont il cherche à se défaire grâce à ce sacrifice. "Celui qui est dit sacer", écrit Benveniste, "porte une véritable souillure qui le met hors de la sociétê des hommes : on doit fuir son contact" (16). L'ordre du sacré, chez les Massa, n'est pas sans rappeler la conception latine. La parole du sacrifiant mettrait son interlocuteur sous l'emprise dangereuse de la divinité. L'homme qui se trouve désormais menacé par la puissance propitiée devra, à son tour, lui dédier un sacrifice.

Le sacrifiant, soumis à des régles d'évitement, comme le sont 1e meurtrier et sa victime est, 1 i aussi, 1a.proie d'une puissance qui le rend malade lui ou sa famille. Le sacrifice massa doit établir une coupure, une distance entre la puissance et le sacrifiant. Ainsi, lorsqu'un homme porte en brousse, au bord de l'eau ou à la croisée des chemins, le sacrifice qu'il dédie au génie du lieu, sortant 1 'animal ou les objets sacrificiels de l'enclos pour en marquer la provenance et pour les introduire dans le territoire de la puissance propitiée, il établit, par le biais de cette "re-territorialisation", une coupure avec le gênie qui, acceptant l'offrande, ne doit plus revenir chez lui. Les sacrifices individuels, effectués à la suite de maux divers, sont en effet destinés à repousser la puissance qui cherche à atteindre le sacrifiant. Si, dans le cas cité, le parcours et l'offrande permettent cette sêparation, pour le sacrifice réalisé sur la tombe,

(16) E. Benveniste, Le vocabulaire des institutions indo-européennes, Paris, Ed. de Minuit, 1969, vol. II, p. 189. 
après. I'enterrement, c'est le sang de la victime qui opère une sorte d'isolat territorial : la tombe, située au sein de l'enclos, devant 1 'entrêe de la case du dêfunt, sera séparée du reste de l'habitation par le sang d'un mouton sacrificiel (sacrifice de type porra) qui, s'écoulant à terre, démarque l'emplacement du mort de celui des vivants. Dans le cas d'un noyé (17), le corps ne pouvant être inhumé sur le lieu d'habitation, on le restitue à son territoire par le biais d'un élément qui marque sa présence; l'ayant ainsi réintroduit, on opère, comme à chaque rite funéraire, une coupure grâce au sang sacrificiel qui isole la tombe du reste de 1'enclos.

Reprenons l'examen de l'enjeu en cours dans le sacrifice qu'accomplit le meurtrier. En portant en brousse un couple de chiots et un oeuf, le tueur remet entre les mains de Matna ce qu'il veut échanger contre sa propre vie et son pouvoir de procréation. La prière explicite largement le sens de cet enjeu : "Matna, reçois ces choses, prends-les à la place de mon propre corps, préserve ma descendance et donne-nous la fraîcheur". Le pouvoir vengeur de sa victime, manoeuvré par Matna, met en danger plus que sa vie : son pouvoir de procréation et sa descendance. La vie même du meurtrier représente bien peu eu égard à l'extermination d'une lignée. Le tokora atteint donc ce qui est le plus cher aux yeux des Massa : ses descendants. Sans fils pour lui offrir du sang sacrificiel après sa mort, le meurtrier ne sera qu'un ancêtre abandonné. Dans le seul but d'épargner ses enfants, un homme qui a battu sa femme à mort ne se pliera pas au rituel du tokora, si elle n'est pas morte sur le coup. Il agira comme si elle avait succombé des suites d'un accident.

Le sacrifice doit annuler les effets du tokora qui s'attaque au pouvoir de procréation du meurtrier, 1 'instance invoquée au cours de chaque rituel sacrificiel vise à rétablir et à augmenter la procréation du sacrifiant. Le sacrifice auquel on recourt pour l'obtention d'une riche descendance apparaît donc comme antinomique du tokora qui extermine les enfants du meurtrier. Ce point essentiel nous permet sans doute de mieux appréhender la portée du choix des victimes sacrificielles

(17) Cf. plus haut 
opêré par les Massa. En prenant soin de mettre à mort des victimes sacrificielles dénuées de force vengeresse, les Massa révèlent leur souci de dissocier le sacrifice du crime et de se protéger des conséquences du crime par le sacrifice. Que le chien, animal le plus proche de 1 'homme, soit exclu du sacrifice sanglant fait apparaître le sacrifice comme une forme de "dêni" de meurtre, les Massa cherchant à gomer la violence d'une mort sanglante rappelant celle de 1 'homicide.

La représentation du crime dévoile la relation symbiotique entre le meurtrier et sa victime et permet de mettre en lumière les liens qui. unissent un individu à sa victime sacrificielle. Dans un prochain article, nous nous interrogerons sur les fondements de l'efficacité de la mort sacrificielle : 'Pourquoi, comme le demande Benveniste, 'sacrifier' veut-il dire en fait 'mettre à mort' quand il signifie proprement 'rendre sacré'" ? L'examen du schéma sacrificiel dans son rapport aux opérations de pensée fournira $I^{\prime}$ axe de cette ëtude. Le sacrifice qui opère comme le langage par substitution et par perte peut apparaître comme une dramatisation du processus de signification qui pose l'home comme producteur de signes et dépendant d'un sens qui le domine. La représentation de la mort est coextensive à la pensée : la cosmogonie dogon dit que la mort a été donnée aux honmes en même temps que la parole. La mort n'existe, en effet, que pour un être capable de se reprêsenter 1 'absence, qui a sẻparé les choses de leur représentation. Si "toute compréhension conceptuelle êquivaut à un meurtre", comme l'écrit Hegel, la pratique sacrificielle peut s'analyser comme la maîtrise provisoire de la vie en tant que redoublement et reprise de la matrice significative qui justifie la substitution du symbole au symbolisé (de la victime au sacrifiant) et qui fonde sa destruction comme productrice à la fois de sens et de vie. La constatation que le redoublement sacrificiel de cette matrice soit une reprise, que la production de sens soit aussi production de vie nous permettra d'examiner une constante de la pratique sacrificielle : une appropriation d'homme. 